\title{
Finance for nutrition
}

\author{
The 'toxic cocktail' of the COVID-19 pandemic, climate and conflict has slowed progress on nutrition. Where \\ long-standing budgets for nutrition have been constrained in these times, broad stakeholder engagement and \\ non-traditional approaches to nutrition financing are needed.
}

M idway through the Decade of Action on Nutrition, and in the final month of the Year of Action on Nutrition, Japan has hosted the third Nutrition for Growth Summit in Tokyo. Nutrition for Growth is described as a 'moment' that enables governments, donors, civil society and the private sector to pledge financially and commit to impactful action on ending malnutrition in all its forms. Framing the challenges of nutrition within health, food and geopolitical systems, Nutrition for Growth 2021 drew political and financial commitments on tackling malnutrition through investment in health systems, climate-smart sustainable food systems and building resilience in conflict-affected regions.

Progress on nutrition is blighted by what's been described as a 'toxic cocktail' of the COVID-19 pandemic, climate change and conflict. The scale of global malnutrition has been updated by the 2021 Global Nutrition Report ${ }^{1}-149.2$ million children under 5 are stunted, 45.4 million are wasted, 28.9 million are overweight; 2.2 billion adults are overweight or obese and $30 \%$ of women of childbearing age suffer from anaemia. At an official Nutrition for Growth side-event, Hungry for Change: Food Systems for Nutrition and Health, hosted by Nature Food and Springer Nature, the Standing Together for Nutrition consortium estimated that the COVID-19 crisis will, over the next 3 years, cause an additional 3.5 million children to suffer stunting, 13.6 million children to suffer wasting and an additional 283,000 deaths related to malnutrition in under-5s in low- and middle-income countries. The burden of malnutrition in all its forms is not equitably distributed around the world. These numbers and their global distribution elicit intense reflections on morality and social justice, but are most often discussed in the pragmatic language of finance - how much investment is needed, who will invest and through what mechanisms?

Estimates for the investments needed to achieve Sustainable Development Goal 2, Zero Hunger, are shaped by a variety of definitions, assumptions, objectives and strategies - including acceptable levels of hunger, direct or indirect interventions, actions that are nutrition-specific or nutrition-sensitive. In 2017, the World Bank priced the achievement of the World Health Assembly Targets (40\% reduction in child stunting, $50 \%$ reduction in anaemia in women; $50 \%$ increase in exclusive breastfeeding rates, child wasting reduced to $5 \%)$ through targeted nutrition interventions at an additional US\$7 billion, minimum, per year to $2025^{2}$. This has been revised upwards to an additional annual investment in nutrition interventions of US $\$ 10.8$ billion to $2030^{1}$; in addition to US $\$ 39-50$ billion annually for nutrition-sensitive financing of obesity prevention policies ${ }^{3}$.

With the brief of ending hunger, earlier this year an exercise by researchers at the University of Bonn examined the best investment options ${ }^{2}$. Lifting 500 million people out of hunger by 2030 - a G7 target - would require investment of US\$11-14 billion per year. Their analysis shows the outcomes could be achieved with least cost through agricultural research and development efficiency enhancement, agricultural extension services, information and communications technology, agricultural information services, small-scale irrigation expansion in Africa, female literacy improvements and scaling up existing social protection. Investment of US \$39-50 billion per year could lift 840 million-909 million people out of hunger by $2030^{2}$. Ceres 2030 models indicate that an additional US $\$ 33$ billion per year to 2030 is needed to end hunger, through doubling the income of small-scale farmers while limiting agricultural emissions in line with the Paris Agreement ${ }^{4}$.

Prevalence of and investment needed for malnutrition may vary between studies, but they are largely quantifiable. Member states at Nutrition for Growth 2021 have outlined comprehensive national strategies to monitor and reduce the burden of malnutrition. Data are key to inform those strategies. Commitments were clearly expressed. Yet, the double economic and health shock of the pandemic has constrained domestic and international resources, and traditional financing for nutrition is at risk of deprioritization. The 2021 Global Nutrition Report has called for non-traditional sources of finance - including the private sector and venture capital investors - to fill the gap in the short to medium term ${ }^{1}$.

Although small- and medium-sized businesses, which make up a half of enterprise within food systems, have been hailed as 'vanguards of a revolution' in the UN Food Systems Summit, there are, at times, uneasy relationships between the food and nutrition community and the private sector. Growing corporate concentrations and power in agrifood systems, eroded trust ${ }^{5}$, and the infiltration of highly processed foods in diets around the world are contentious issues. Yet, there are opportunities for the food and nutrition communities - academics, analysts, practitioners - to foster shared objectives within the private sector. Nutrition for Growth 2021 outlined mechanisms for greater responsibility within the food industry for nutrition governance, reformulation of foods, responsible marketing, and improving nutrition in the workplace and workforce. Mechanisms for accountability on pledges is key, but so too is an environment that guides and supports businesses towards sustainably delivering for nutrition.

In a World View article in this issue of Nature Food, Samir Kaul of Khosla Ventures provides insights into how venture capital can accelerate food technology innovation to support healthy, sustainable diets. Venture capitalists motivated by addressing intractable problems in society are increasingly prepared to take big risks in the food sector - can these ventures be used to alleviate malnutrition?

Moral and social justice motivations cannot be dismissed from conversations about serious finance. Effective altruism — or direct giving - as proposed by Peter Singer ${ }^{6}$ has innovated with the times. Mobile and digital technologies have made the world much smaller, removing distance between the act of giving and evidence of impact - between the giver and beneficiary. Direct giving seems to facilitate a favourable 'bang for buck' - and indeed, the mechanism has found appeal with digital philanthropists. Can this willingness to give be harnessed for alleviating malnutrition? 
And so, just as the COVID-19 pandemic has changed how we do business in so many ways, the 2021 Global Nutrition Report is calling for a new way of doing business with regard to financing nutrition. Nutrition for Growth has the potential to pull a diverse collection of stakeholders together - policymakers, donors, businesses, scientists - for a mosaic approach to financing nutrition and facilitating multisectoral, collective action. There may be additional opportunities for the nutrition community to motivate non-traditional sources of finance by creating supportive environments for all stakeholders, sharing technical and political expertise, and also by combining the pragmatic language of finance with the universal language of social impact and giving.

Published online: 16 December 2021 https://doi.org/10.1038/s43016-021-00446-y
References

1. 2021 Global Nutrition Report: The State of Global Nutrition https:// go.nature.com/3EgBKSk (Development Initiatives, 2021).

2. Chichaibelu, B. B., Bekchanov, M., von Braun, J. \& Torero, M. Food Policy 104, 102151 (2021).

3. Achieving Zero Hunger: The Critical Role of Investments in Social Protection and Agriculture https://go.nature.com/32THtj7 (FAO, 2015).

4. Laborde, D., Murphy, S., Parent, M., Porciello, J. \& Smaller C. Ceres2030: Sustainable Solutions to End Hunger-Summary Report https://go.nature.com/3oc32n7 (Cornell University, IFPRI and IISD, 2020).

5. Yates, J., Gillespie, S., Savona, N., Deeney, M. \& Kadiyala, S. BMJ Glob. Health 6, e007350 (2021).

6. Singer, P. Phil. Public Affairs 1, 229-243 (1972). 